\title{
Computationally efficient nonlinear Froude-Krylov force calculations for heaving axisymmetric wave energy point absorbers
}

\author{
Giuseppe Giorgi $^{1}{ }^{(1)} \cdot$ John V. Ringwood $^{1}$
}

Received: 17 February 2016 / Accepted: 20 July 2016 / Published online: 29 July 2016

(C) Springer International Publishing Switzerland 2016

\begin{abstract}
Most wave energy converters (WECs) are described by linear mathematical models, based on the main assumption of small amplitudes of motion. Notwithstanding the computational convenience, linear models can become inaccurate when large motions occur. On the other hand, nonlinear models are often time consuming to simulate, while model-based controllers require system dynamic models which can execute in real time. Therefore, this paper proposes a computationally efficient representation of nonlinear static and dynamic Froude-Krylov forces, valid for any heaving axisymmetric point absorber. Nonlinearities are increased by nonuniform WEC cross sectional area and large displacements induced by energy maximising control strategies, which prevent the device from behaving as a wave follower. Results also show that the power production assessment realized through a linear model can be overly optimistic and control parameters calculations should also reflect the true nonlinear nature of the WEC model.
\end{abstract}

Keywords Wave energy - Axisymmetric point absorber . Linear potential theory $\cdot$ Nonlinear Froude-Krylov force . Latching control

\section{Introduction}

In wave energy applications, the accuracy of the mathematical model of a wave energy device is crucial to simulate the

Giuseppe Giorgi

ggiorgi@eeng.nuim.ie

John V. Ringwood

john.ringwood@eeng.nuim.ie

1 Centre for Ocean Energy Research, Maynooth University, Kildare, Ireland correct motion and to determine a reliable power production assessment, as well as to design model-based controllers, which depend, either explicitly or implicitly, on the mathematical model of the system to determine the control parameters. Usually, the model is linear and based on Cummins equation (Cummins 1962), with hydrodynamic parameters calculated using boundary element methods (BEMs). Notwithstanding the simplicity and the computational convenience of a linear model, the hypotheses under which the linear model is valid are quite restrictive, in particular the assumption of small motion.

Indeed, the purpose of a wave energy converter (WEC) is to exaggerate the motion to maximize the power production. As a result, nonlinearities become important and linear models become relatively inaccurate. Several studies have shown significant differences between linear models and experimental tank tests (Babarit et al. 2009), fully nonlinear models like CFD (Giorgi and Ringwood 2016a), or partially nonlinear models (Merigaud et al. 2012).

While the computational effort usually increases considerably with the number of nonlinearities included in the model, the gain in accuracy depends on the relevance of each nonlinear effect on the particular device (Peñalba et al. 2015a). For the heaving point absorber in Fig. 2, the most relevant nonlinear component of the hydrodynamic force is the Froude-Krylov (FK) force (Merigaud et al. 2012), which is the integration of the incident pressure over the wetted surface.

While, in the linear approach, the FK force is computed over the constant mean wetted surface, in a nonlinear approach the pressure is integrated over the instantaneous wetted surface, which requires a significant additional computational effort, since it implies the usage of either a very fine mesh (Babarit et al. 2009) or an automatic remeshing routine of the surface (Gilloteaux 2007). These nonlinear solutions 
return accurate results but at a computational price too high to be compatible with the real-time simulation required by model-based controllers. This paper proposes a computationally efficient algebraic calculation of the nonlinear FK force valid for axisymmetric heaving point absorbers. Note that such devices normally satisfy the condition of rotational symmetry and the algebraic equations derived here can be applied both to single and multi-body devices, when the hydrodynamic interaction between bodies does not deform the incident wave significantly.

A purely algebraic calculation of nonlinear FroudeKrylov forces is achievable for axisymmetric point absorbers constrained to move in heave only, as the WAVESTAR device (Hansen and Kramer 2011), or for axisymmetric point absorbers operating mainly in waves long with respect to the device diameter, as the pitch motion is negligible. In case of combined heave and pitch motion, no algebraic solution exists and the FK integral has to be solved numerically.

The focus of the paper is on WECs operating in the power production region, using a model-based control system to maximise power capture. The vast majority of waves in the power production region are linear, though significant nonlinear WEC dynamics may be excited. In contrast, many studies (Henry et al. 2013; Rafiee and Dias 2013) utilise (computationally complex) nonlinear WEC simulations to assess the impact of extreme waves, usually using computational fluid dynamics (CFD) (Agamloh et al. 2008) or smooth particle hydrodynamics (SPH) (Omidvar et al. 2012). This paper targets nonlinear wave-body interactions caused by linear waves in the power production region only. Therefore, the paper does not purport to address the area of WEC device survivability or extreme loading because such sporadic events, which are not significant for the power production assessment, cannot be described by linear wave theory.

This paper implements and compares three different methods:

(a) A linear hydrodynamic model,

(b) A nonlinear Froude-Krylov model, using a remeshing routine to compute the instantaneous wetted surface,

(c) A nonlinear Froude-Krylov model, using the algebraic solution of the Froude-Krylov force integral.

The linear, remeshing and algebraic approaches are used to simulate the motion of a heaving sphere with centroid at the still water level under linear monochromatic waves, to highlight the nonlinearities deriving only from the FK force at each individual wave frequency. The chosen geometry is a sphere, which has a cross-sectional area (CSA) which varies with the instantaneous draft of the device, which is likely to emphasize the nonlinearity of the FK force. To maximize the power production in each sea state, latching control has been implemented. Latching is a discrete controller that locks the device motion at its extrema for an appropriate latching duration so that the amplitude of motion is exaggerated (Budal et al. 1979). As a consequence, the wetted surface experiences greater changes, exaggerating the relevance of nonlinearities. The performance of methods (a), (b) and (c) (linear, remeshing nonlinear Froude-Krylov and algebraic nonlinear Froude-Krylov) is compared in terms of amplitude of motion, optimal control parameters, power production and computational time.

The reminder of the paper is organized as follows: Sect. 2 presents the theoretical background on which the modelling approaches described in Sect. 3 are based. Section 4 presents, in detail, the algebraic solution of the nonlinear FK force integral. A case study is analyzed in Sect. 5 and results are given in Sect. 6. Some conclusions and final remarks are presented in Sect. 7.

\section{Theoretical background}

The fluid is assumed inviscid and the incident flow irrotational and incompressible. The right-handed inertial reference frame is centered at the hydrostatic equilibrium position of the body, which is coincident with the gravity center. Newton's second law can be used to describe the system dynamics as follows:

$$
m \ddot{\boldsymbol{\xi}}(t)=\mathbf{F}_{\mathbf{g}}-\iint_{S(t)} P(t) \mathbf{n} \mathrm{d} S+\mathbf{F}_{\mathrm{PTO}}(t)
$$

where $m$ is the mass of the body, $\boldsymbol{\xi}=(x, y, z)$ the general displacement of the body from its hydrostatic equilibrium position, $\mathbf{F}_{\mathbf{g}}$ the gravity force, $S$ the submerged surface, $P$ the pressure, $\mathbf{n}$ a vector normal to the surface and $\mathbf{F}_{\text {PTO }}$ the power take-off force.

The pressure $P$ can be derived from the incident flow applying Bernoulli's equation:

$P(t)=-\rho g z(t)-\rho \frac{\partial \phi(t)}{\partial t}-\rho \frac{|\nabla \phi(t)|^{2}}{2}$

where $\rho$ is the water density, $g$ the acceleration of gravity, $P_{\mathrm{st}}=-\rho g z$, hydrostatic pressure and $\phi$ the potential flow, which can be decomposed as the sum of the undisturbed incident flow potential $\phi_{\mathrm{I}}$, the diffraction potential $\phi_{\mathrm{D}}$ and the radiation potential $\phi_{\mathrm{R}}$ :

$\phi(t)=\phi_{\mathrm{I}}+\phi_{\mathrm{D}}+\phi_{\mathrm{R}}$

Combining Eqs. (1-3), different forces can be defined:

- $\mathbf{F}_{F K_{s t}}$ is the static Froude-Krylov force, given as the balance between the gravity force and the Archimedes force: 


$$
\mathbf{F}_{F K_{s t}}(t)=\mathbf{F}_{g}-\iint_{S(t)} P_{s t}(t) \mathbf{n} \mathrm{d} S
$$

- $\mathbf{F}_{F K_{d y}}$ is the dynamic Froude-Krylov force:

$$
\mathbf{F}_{F K_{d y}}(t)=-\iint_{S(t)} P_{d y}(t) \mathbf{n} \mathrm{d} S
$$

where $P_{d y}=-\rho \frac{\partial \phi_{I}}{\partial t}-\rho \frac{\left|\nabla \phi_{I}\right|^{2}}{2}$ the dynamic pressure.

$-\mathbf{F}_{D}$ is the diffraction force:

$$
\mathbf{F}_{D}(t)=-\iint_{S(t)} P_{D}(t) \mathbf{n} \mathrm{d} S
$$

where $P_{D}=-\rho \frac{\partial \phi_{D}}{\partial t}-\rho \frac{\left|\nabla \phi_{D}\right|^{2}}{2}$ the diffraction pressure. $-\mathbf{F}_{R}$ is the radiation force:

$$
\mathbf{F}_{R}(t)=-\iint_{S(t)} P_{R}(t) \mathbf{n} \mathrm{d} S
$$

where $P_{R}=-\rho \frac{\partial \phi_{R}}{\partial t}-\rho \frac{\left|\nabla \phi_{R}\right|^{2}}{2}$ the diffraction pressure.

The time-dependance annotation will be omitted for brevity hereafter. Using Eqs. (4-7), (1) can be rewritten as:

$$
m \ddot{\xi}=\mathbf{F}_{F K_{s t}}+\mathbf{F}_{F K_{d y}}+\mathbf{F}_{D}+\mathbf{F}_{R}+\mathbf{F}_{P T O}
$$

Note that, since the fluid is assumed to be inviscid, no viscous force appears in (8). However, a viscous term could be added in (8), for example using the Morrison equation (Morison et al. 1950):

$\mathbf{F}_{v i s}=-\frac{1}{2} \rho C_{d} A_{d}\left|\mathbf{V}-\mathbf{V}_{\mathbf{0}}\right|\left(\mathbf{V}-\mathbf{V}_{\mathbf{0}}\right)$

where $C_{d}$ is the drag coefficient, $A_{d}$ is the characteristic surface, $V$ is the velocity of the floater and $V_{0}$ is the undisturbed flow velocity.

\section{Modelling approaches}

The formulation proposed in Sect. 2 presents some sources of nonlinearities: Bernoulli's Eq. (2) has quadratic terms, the incident potential flow can be nonlinear and the wetted surface may vary in time. While the quadratic terms in (2) are assumed negligible and only linear waves are considered, the wetted surface is modelled as either constant or variable, leading respectively to a linear or nonlinear representation of Froude-Krylov forces, resulting in linear and nonlinear Froude-Krylov hydrodynamic models. Radiation and diffraction forces are assumed to be linear in both models: Falnes (2002) shows that diffraction is negligible when the device dimension is considerably smaller than the wave length. Likewise, Clement and Ferrant (1988) show that radiation nonlinearities are negligible for floating bodies small compared to the wavelength. Merigaud et al. (2012) implemented both nonlinear Froude-Krylov and nonlinear radiation and diffraction forces for a heaving point absorber, showing that the response of the device is mainly affected by nonlinear FK forces, while nonlinear radiation and diffraction forces have minor effects on the system dynamics. Furthermore, Gilloteaux (2007) and Guerinel et al. (2013) use real tank experiments, with the SEAREV device and the WAVESTAR device respectively, to validate a nonlinear Froude-Krylov model with linear radiation and diffraction term, showing a significant improvement with respect to a fully-linear model and good agreement with the experimental measurement. Similar results are obtained by Giorgi and Ringwood (2016b), who compare a nonlinear FroudeKrylov model which includes a viscous term with a fully nonlinear CFD model.

\subsection{Linear model}

The linear approach assumes a small amplitude and steepness of the wave, thus the potential problem is linearized and solved around the equilibrium position of the device. Under the linear assumption, the mean wetted surface $S_{M}$ is used and Eq. (8) becomes:

$$
\begin{aligned}
m \ddot{\boldsymbol{\xi}}=\underbrace{-K_{H} \xi}_{F_{F K_{S t}}}-\underbrace{\int_{-\infty}^{\infty} K_{e x}(t-\tau) \eta(\tau) \mathrm{d} \tau}_{\mathbf{F}_{e x}=\mathbf{F}_{F} K_{d y}+\mathbf{F}_{D}} \\
-\underbrace{\mu_{\infty} \ddot{\boldsymbol{\xi}}-\int_{-\infty}^{\infty} K_{R}(t-\tau) \dot{\boldsymbol{\xi}}(\tau) \mathrm{d} \tau}_{\mathbf{F}_{R}}-\mathbf{F}_{P T O}
\end{aligned}
$$

where:

- $\mathbf{F}_{F K_{s t}}$ is described by the hydrostatic stiffness $K_{H}$;

- $\mathbf{F}_{e x}$ is represented by the convolution product between the excitation impulse-response function (IRF) $K_{e x}$ and the free-surface elevation $\eta$;

- $\mathbf{F}_{R}$ is represented by the added mass $\mu_{\infty}$ and the convolution product between the radiation IRF $K_{R}$ and the velocity $\dot{\xi}$, based on Cummins equation (Cummins 1962);

The added mass and the impulse response functions are calculated by the time domain BEM software ACHIL3D (Babarit 2010). The radiation convolution, which is computationally expensive to compute directly, is replaced by its state space representation (Taghipour et al. 2007). 


\subsection{Nonlinear Froude-Krylov force model}

While the linear model uses the constant mean wetted surface $S_{M}$, the nonlinear FK model computes the Froude-Krylov forces considering the exact instantaneous wetted surface $S(t)$, namely integrating the fluid pressure over the actual submerged portion of the body, as it moves through the water:

$\mathbf{F}_{F K}=\mathbf{F}_{F K_{s t}}+\mathbf{F}_{F K_{d y}}=\mathbf{F}_{g}-\iint_{S(t)}\left(P_{s t}+P_{d y}\right) \mathbf{n} \mathrm{d} S$

Note that both static and dynamic Froude-Krylov forces depend on the instantaneous wetted surface, which depends both on the incident wave elevation and the displacement of the body. The remaining force components of (8) are computed linearly: the radiation force is described by Cummins equation as in (10) while the diffraction force is represented by the convolution product between the diffraction impulseresponse function $K_{7}$ and the free surface elevation $\eta$ :

$\mathbf{F}_{D}=-\int_{-\infty}^{\infty} K_{7}(t-\tau) \eta(\tau) \mathrm{d} \tau$

Note that, while in the linear model the diffraction force is computed together with the dynamic Froude-Krylov force by means of the excitation IRF $K_{e x}$, in the nonlinear FK model the diffraction force is calculated independently through the diffraction IRF $K_{7}$.

For a general geometry of the device, the computation of the integral in (11) is performed by discretizing the surface of the body with a finite mesh and summing up the forces generated by the pressure acting on the area of each plane panel. At each time step, as the body moves, an automatic (computationally expensive) routine re-meshes the partially submerged panels around the free surface and updates the instantaneous wetted surface. A detailed description of the remeshing routing can be found in Gilloteaux (2007).

In contrast, this paper shows an algebraic solution to the pressure integral in (11), valid for any axisymmetric geometry subject to deep water linear waves, achieving the same results of the remeshing routine but with a considerable computational saving, as will be shown in Sect. 6. The details of the pressure integral calculations are provided in Sect. 4.

\section{Algebraic solution}

The algebraic calculation of the integral in (11) requires the explicit definition of the pressure $P$, the infinitesimal surface element $\mathbf{n} \mathrm{d} S$ and the limits of integration. The pressure is obtained applying Airy's wave theory for deep water waves:
$P(x, z, t)=\rho g a \mathrm{e}^{\chi z} \cos (\omega t-\chi x)-\rho g z$

where $x$ is the direction of wave propagation, $z$ is the vertical direction (positive upwards), $a$ is the wave amplitude, $\chi$ the wave number and $\omega$ the wave frequency.

The solution of the pressure integral is presented for two different types of axisymmetric devices: Sect. 4.1 considers point absorbers constrained to heave only, for which an algebraic solution is attainable, while Sect. 4.2 presents the formulation for point absorbers moving in both heave and pitch directions, for which a numerical solution of the integral is required.

\subsection{Heaving point absorber}

As shown in Fig. 1, the geometry is assumed axisymmetric with a fixed vertical axis, so it is possible to describe its surface in parametric cylindrical coordinates:

$\left\{\begin{array}{l}x(\sigma, \theta)=f(\sigma) \cos \theta \\ y(\sigma, \theta)=f(\sigma) \sin \theta, \quad \theta \in[0,2 \pi) \wedge \sigma \in\left[\sigma_{1}, \sigma_{2}\right] \\ z(\sigma, \theta)=\sigma\end{array}\right.$

Table 1 shows some examples of profiles of revolution $f(\sigma)$ using the cylindrical coordinates in (14).

Considering the canonical basis given by the radial and tangent unit vectors, $\mathbf{e}_{\sigma}$ and $\mathbf{e}_{\theta}$ respectively, the infinitesimal surface element $\mathrm{d} S$ becomes:

$\mathbf{n} \mathrm{d} S=\mathbf{n}\left\|\mathbf{e}_{\sigma} \times \mathbf{e}_{\theta}\right\| \mathrm{d} \sigma \mathrm{d} \theta=\mathbf{n} f(\sigma) \sqrt{f^{\prime}(\sigma)^{2}+1} \mathrm{~d} \sigma \mathrm{d} \theta$

Finally, the point absorber is constrained to move only in heave (along the unit vector $\mathbf{k}$ of the $z$-axis), therefore, only the vertical component $P_{z}$ of the pressure is taken into account:

$P_{z}=P \cdot\langle\mathbf{n}, \mathbf{k}\rangle=\left\langle\frac{\mathbf{e}_{\sigma} \times \mathbf{e}_{\theta}}{\left\|\mathbf{e}_{\sigma} \times \mathbf{e}_{\theta}\right\|}, \mathbf{k}\right\rangle=P \cdot \frac{f^{\prime}(\sigma)}{\sqrt{f^{\prime}(\sigma)^{2}+1}}$

Combining Eqs. (13-16) with (11), the magnitude of the Froude-Krylov force in the vertical direction becomes:

$$
\begin{aligned}
F_{F K_{z}}= & \int_{0}^{2 \pi} \int_{\sigma_{1}}^{\sigma_{2}} P(x(\sigma, \theta), z(\sigma, \theta), t) f^{\prime}(\sigma) f(\sigma) \mathrm{d} \sigma \mathrm{d} \theta \\
= & \int_{0}^{2 \pi} \int_{\sigma_{1}}^{\sigma_{2}}\left(\rho g a \mathrm{e}^{\chi \sigma} \cos (\omega t-\chi f(\sigma) \cos \theta)-\rho g \sigma\right) \\
& \times f^{\prime}(\sigma) f(\sigma) \mathrm{d} \sigma \mathrm{d} \theta
\end{aligned}
$$

Referring again to Fig. 1, with $h_{0}$ the draft at equilibrium, $z_{d}(t)$ the vertical displacement of the body and $\eta(t)$ the free surface elevation of the undisturbed incident flow at $x=0$, 
Fig. 1 Axisymmetric heaving device with generic profile $f(\sigma)$ : the figure on the left shows the rest position, with the center of gravity at the still water level (SWL) and draft $h_{0}$; the figure on the right shows the free surface elevation $\eta$ and the device displacement $z_{d}$ after a time $t^{*}$. The pressure is integrated over the surface between $\sigma_{1}$ and $\sigma_{2}$

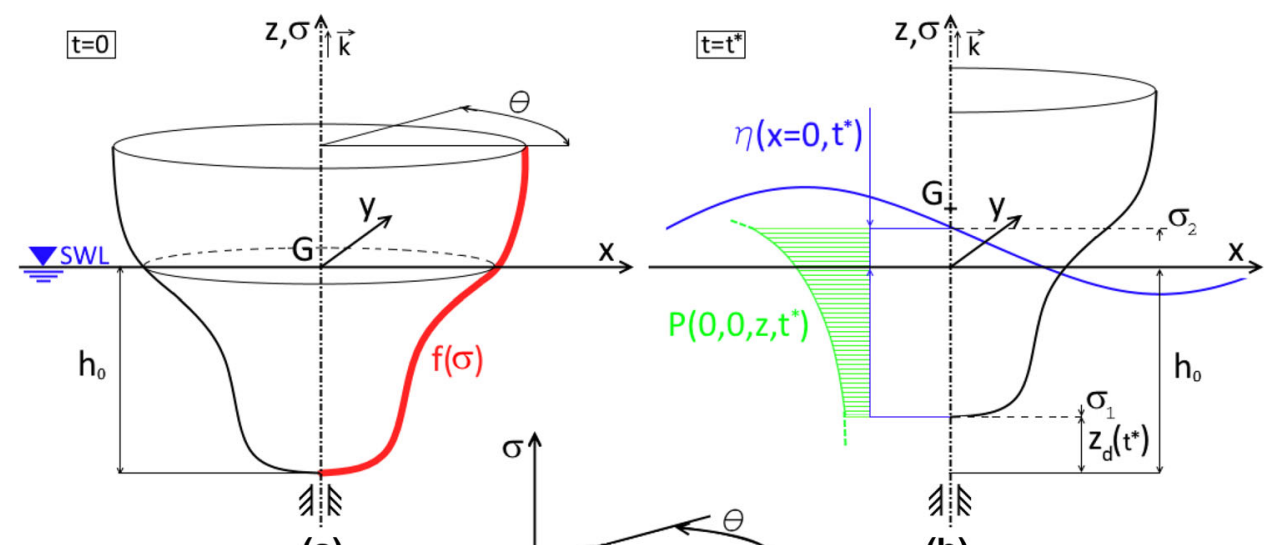

(a) (b) the instantaneous wetted surface is defined by the following limits of integration:

$$
\left\{\begin{array}{l}
\sigma_{1}=z_{d}(t)-h_{0} \\
\sigma_{2}=\eta(t)
\end{array}\right.
$$

The expression for the pressure in (13) is still not directly integrable because of the $x$ dependance in the argument of the cosine. Two solutions are available:

- The long wave approximation, which assumes that the wave length $\lambda$ is considerably longer than the characteristic dimension of the device, so the dependance of the pressure on $x$ is neglected, or

- A McLaurin expansion of the cosine term.

In Sect. 6.1 it will be shown that, while both methods are accurate for long waves, only the McLaurin expansion method (using just three terms of the expansion) is able to return accurate results in every sea state.

The integral in (17) can be solved for any profile $f(\sigma)$ defined either as a polynomial function of order zero (cylinder), order one (cone) or any higher order, as an arc of a circumference (portion of a sphere) or as an exponential. Table 1 summarizes the results for a variety of common geometries under the long wave approximation.

\subsection{Heaving-pitching point absorber}

In this section a two degree of freedom axisymmetric device is considered, where both heave and pitch motions are allowed. Along with the translational force in (11), a resulting torque acts on the body:

$$
\mathbf{T}_{F K}=-\iint_{S(t)}\left(P_{s t}+P_{d y}\right) \mathbf{n} \times \mathbf{r} \mathrm{d} S
$$

where $\mathbf{r}$ is the vector representing the distance from the center of gravity.

Since the body is pitching, the axis of revolution symmetry must rotate as well. Therefore, the cylindrical coordinates of (14) have to be multiplied by the following rotation matrix:

$\mathbf{R}_{\delta}=\left[\begin{array}{ccc}\cos \delta & 0 & \sin \delta \\ 0 & 1 & 0 \\ -\sin \delta & 0 & \cos \delta\end{array}\right]$

where $\delta$ is the pitching angle.

Using pitching cylindrical coordinates, the integrals for heave force in (11) and pitch torque in (19) have no general algebraic solution for axisymmetric point absorbers and a numerical approach is required. Notwithstanding an algebraic solution is not achievable, the numerical integration is a viable option since it is likely to be more computationally efficient than a remeshing routine approach. Nevertheless, this paper aims to deal only with algebraic solution of the FroudeKrylov integral, therefore, purely heaving point absorbers are considered, using the formulation of Sect. 4.1 .

It is worth of notice that a purely pitching prismatic device is suitable to have an algebraic solution for the FroudeKrylov torque. Giorgi and Ringwood (2016c) compare the relevance of nonlinear Froude-Krylov forces, as well as viscous drag, for a heaving point absorber and a bottom-hinged oscillating flap device.

\section{Case study}

The relevance of nonlinear Froude-Krylov forces, hence the difference between the linear and nonlinear FK model, becomes important when the instantaneous wetted surface significantly differs from the mean wetted surface. Two major conditions for this situation follow: 
Table 1 Solution of the integral of the vertical component of static and dynamic pressure under the long wave approximation for axisymmetric devices with different profiles of revolution: vertical line (cylinder), oblique line (cone), arc of circumference (sphere) and exponential profile

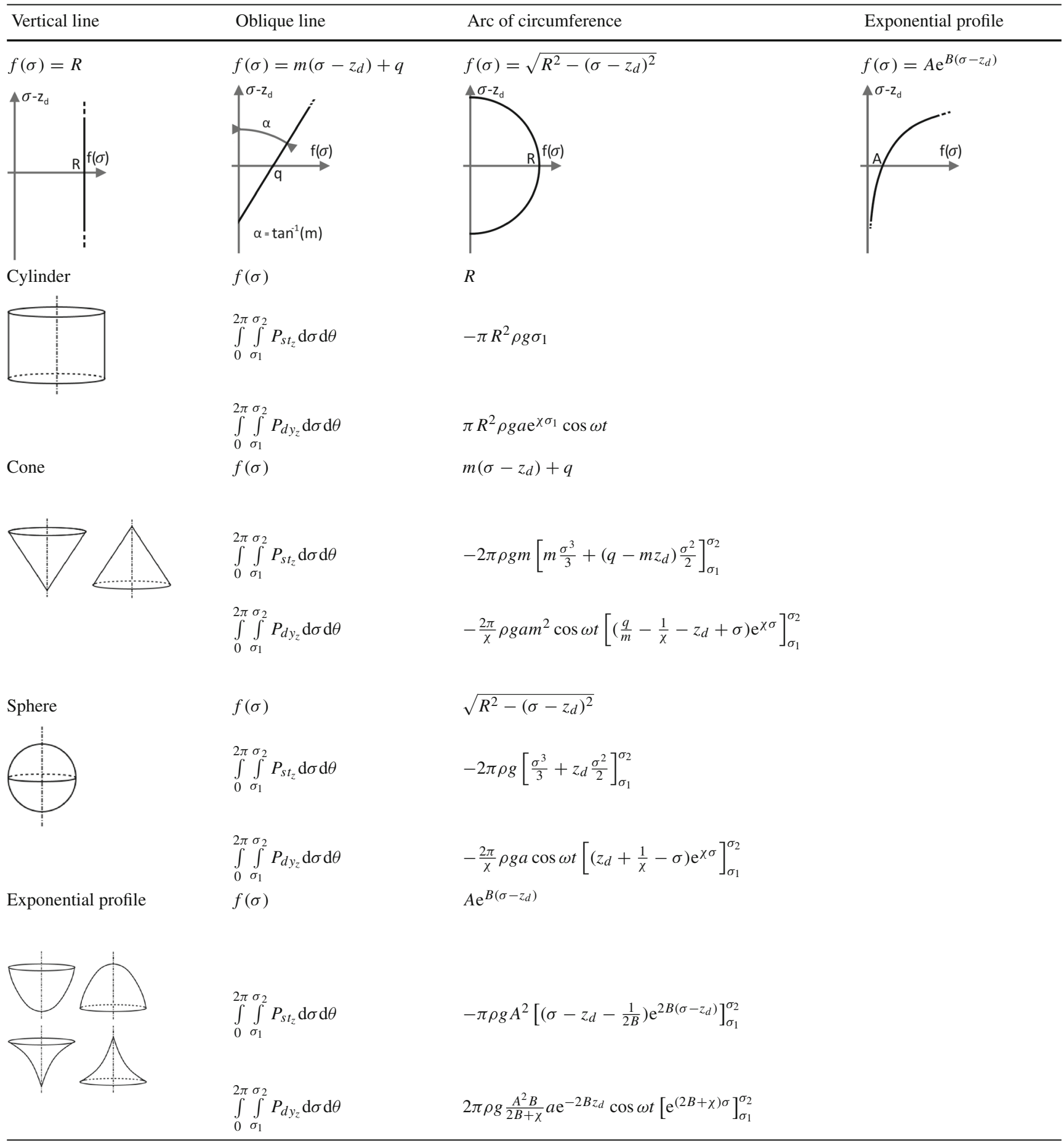

(a) The device has a nonuniform cross sectional area (CSA) and

(b) the device does not behave as a wave follower, namely its displacement $z_{d}$ is significantly different from the wave surface elevation $\eta$.
Note that condition (b) results in significant variations in wetted surface of the WEC. Therefore, as shown in Fig. 2, the device is chosen to be a sphere of $2.5 \mathrm{~m}$ radius, with the gravity center $G$ coincident with the geometric center and a natural period of $3.17 \mathrm{~s}$. The dimension of the sphere is 
Fig. 2 Case study: spherical device (radius $R=2.5 \mathrm{~m}, 3.17 \mathrm{~s}$ natural period $T_{n}$ ) constrained to heave with PTO linear damper and latching mechanism, subject to deep water linear waves

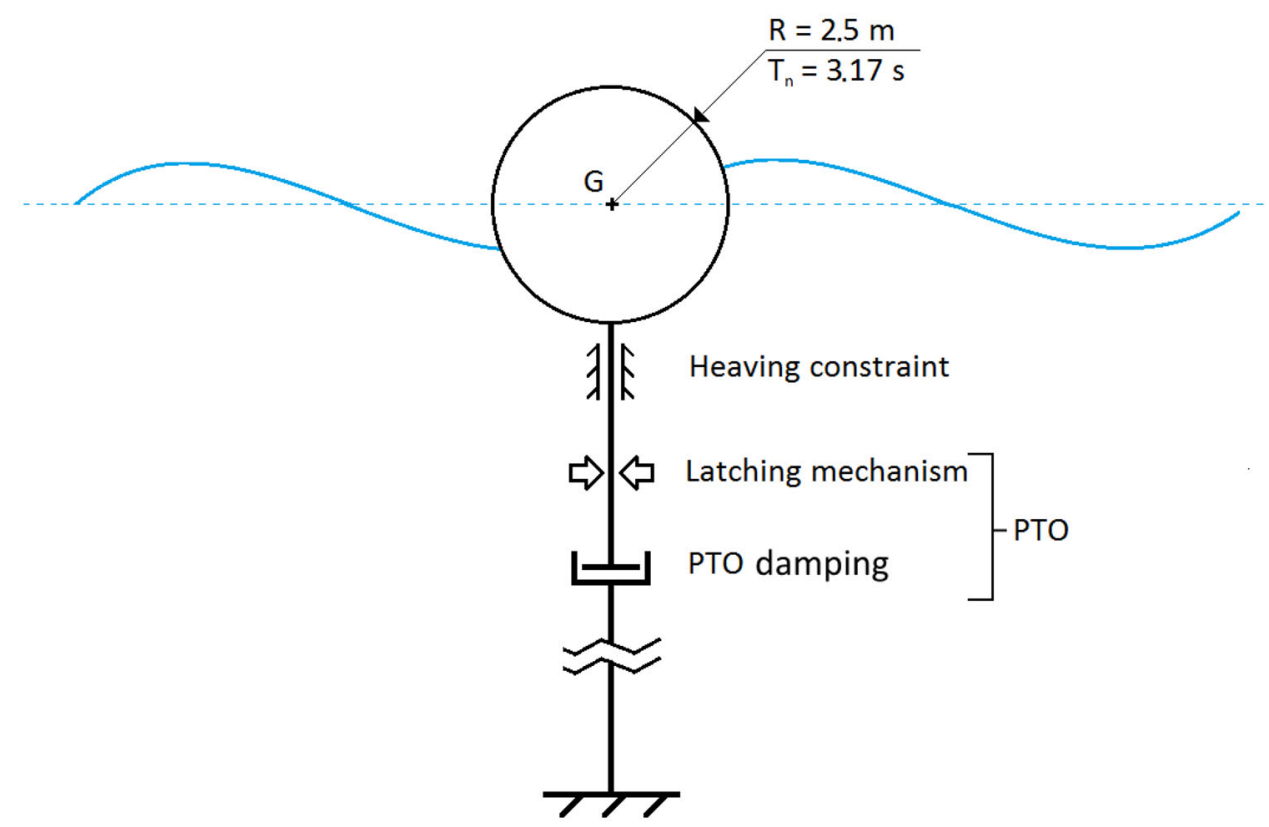

chosen to be comparable with common existing wave energy converters like WAVESTAR (Hansen and Kramer 2011). The device is constrained to heave only and is tethered to the seabed with a linear damper acting as PTO, in combination with a latching mechanism.

To focus only on nonlinearities relative to FK forces, linear waves based on Airy's theory are used, assuming a small steepness $H_{w} / \lambda$ (Mehaute 1976), defined as the ratio between the wave height $H_{w}$ and wave length $\lambda$. The waves are chosen to be monochromatic to analyze the response of the device at each different frequency independently, with wave periods $T_{w}$ chosen to cover the common sea states experienced by a point absorber wave energy device in deep water conditions (Hansen and Kramer 2011). Since FK nonlinearities are caused by changes in the instantaneous wetted surface, which depends on the intersection between the free surface elevation and the body position, the higher the wave steepness, the higher the relevance of FK nonlinearities. Therefore, three different wave steepness indices are considered (0.006, 0.012 and 0.018), where the highest steepness allowed in linear wave theory (Mehaute 1976) generates the most significant nonlinear response. Moreover, the comparison is carried on at constant steepness for different wave periods to have the same (scaled) wave profile and, consequently, a fair comparison. The resulting sea states are summarised in Table 2.

The main objective of a WEC is to maximize the energy captured by the PTO system. Considering the force-tovelocity model of a WEC, in the frequency domain, and for a linear system, Falnes (2002) obtains

$\frac{V(\omega)}{F_{e X}(\omega)+F_{P T O}(\omega)}=\frac{1}{Z_{i}(\omega)}$, where $Z_{i}(\omega)$ is the intrinsic impedance of the system, $V(\omega)$ is the device velocity, $F_{e x}(\omega)$ is the wave excitation force and $F_{P T O}(\omega)$ is the control force. The condition for optimal energy absorption is derived by Falnes (2002) and referred to as complex conjugate control, since the external impedance added by the PTO is required to be the complex conjugate of the intrinsic impedance:

$Z_{P T O}(\omega)=Z_{i}^{*}(\omega)$

Accomplishing the condition in (22) is equivalent to realizing an optimal velocity profile as

$V^{o p t}(\omega)=\frac{F_{e x}(\omega)}{2 R_{i}(\omega)}$

where $R_{i}=1 / 2\left(Z_{i}+Z_{i}^{*}\right)$ is the real part of $Z_{i}$. As a consequence, $R_{i}$ is a real function, so the velocity profile is in phase with the excitation force. Therefore, the optimal complex conjugate control imposes a condition over both the amplitude and the phase of the velocity. A simpler approach is followed by a phase-only control strategy, such as latching, which pursues only the phase matching between the velocity and the exciting force, without considering the amplitude. Latching is a discrete example of phase control, meaning that an on/off PTO force is applied, usually by means of a braking system, to eliminate any phase shift between the velocity and the incoming wave excitation force.

Therefore, a latching control system is implemented to maximize the power capture of the WEC in each sea state. Moreover, especially at frequencies far from resonance, the control system prevents the device behaving like a wave follower, exaggerating the amplitude of motion and, conse- 
Table 2 Sea states: linear monochromatic deep water waves according to Airy's theory
Fig. 3 Latching calculations to put velocity and excitation force in phase. Latching instants at extrema of position $P_{0}$ and $-P_{0}: t_{1}, t_{3}$ and $t_{5}$; latching duration: $T_{L}$; unlatching instants: $t_{2}, t_{4}$ and $t_{6}$

\begin{tabular}{lllllllll}
\hline Wave periods (s) $T_{w}$ & 3 & 4 & 5 & 6 & 7 & 8 & 9 & 10 \\
\hline $\begin{array}{l}\text { Normalized wave lengths, } \lambda / D \\
\text { Wave heights (m), } H_{w}\end{array}$ & 2.8 & 5.0 & 7.8 & 11.2 & 15.4 & 20.0 & 25.2 & 31.2 \\
$\quad$ For $H_{w} / \lambda=0.006$ & 0.08 & 0.15 & 0.23 & 0.34 & 0.46 & 0.60 & 0.76 & 0.94 \\
For $H_{w} / \lambda=0.012$ & 0.17 & 0.30 & 0.47 & 0.67 & 0.92 & 1.20 & 1.52 & 1.87 \\
For $H_{w} / \lambda=0.018$ & 0.25 & 0.44 & 0.69 & 1 & 1.36 & 1.78 & 2.25 & 2.78 \\
\hline
\end{tabular}

Wave lengths have been normalized with respect to the buoy diameter $D=5 \mathrm{~m}$

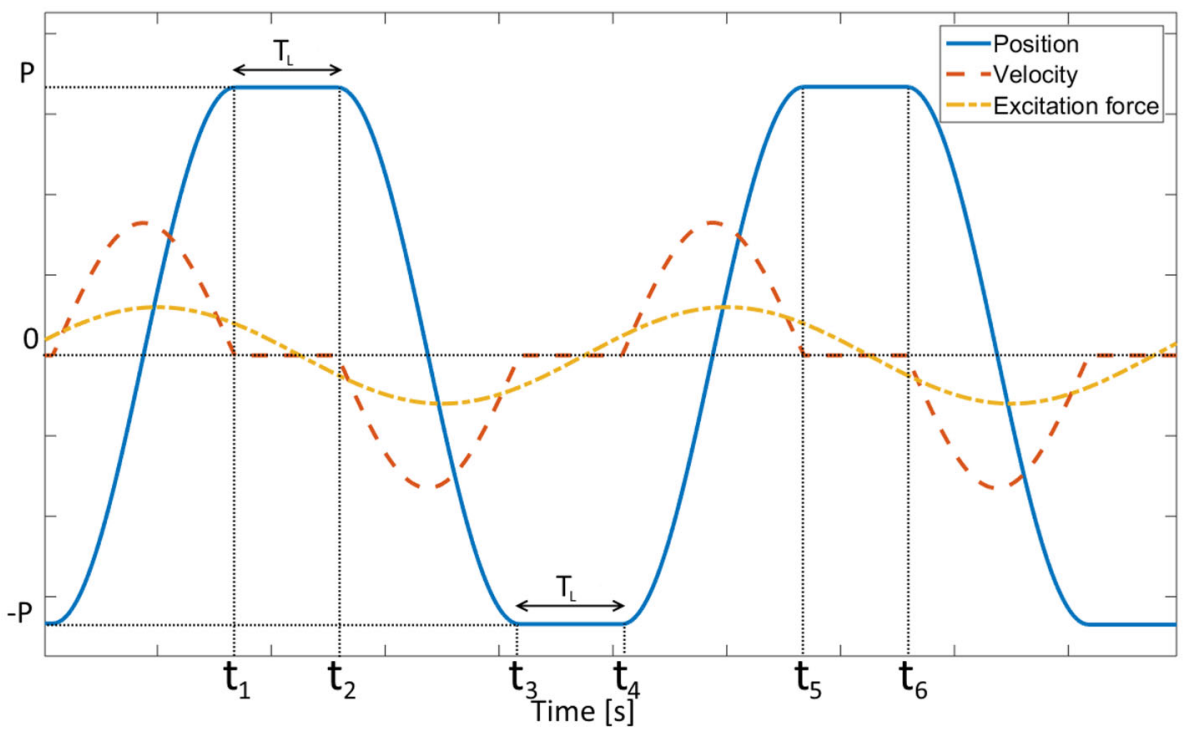

quently, FK nonlinearities (Peñalba et al. 2015b). Latching control is a common solution for point absorbers thanks to its straightforward implementation in a real device and its good performance (Budal et al. 1979; Babarit and Clément 2006).

The objective of power absorption maximization in a linear system is achieved by having the device velocity in phase with the excitation force. Referring to Fig. 3, the device is locked at times $t_{1}, t_{3}$ and $t_{5}$ at the extrema of displacement, namely when the velocity is zero, and released at times $t_{2}, t_{4}$ and $t_{6}$ after a latched duration $T_{L}$.

The control parameters are the PTO damping coefficient $B_{P T O}$ and the latching duration $T_{L}$ which, in a linear case with monochromatic waves, is optimally calculated (Ringwood and Butler 2004) as:

$T_{L}=\frac{t_{5}-t_{1}}{2}-\left(t_{5}-t_{4}\right)=\frac{T_{w}}{2}-\frac{T_{d}}{2}=\frac{T_{w}}{2}-\frac{T_{n}}{2 \sqrt{1-\zeta^{2}}}$

where the damped natural period $T_{d}$ is determined by the natural period $T_{n}$ and the total damping ratio $\zeta$ of the system which, in turns, depends on the sum of the frequencydependent radiation damping and PTO damping.

In the nonlinear FK model, since the cross sectional area is changing during the motion, the hydrostatic stiffness and, consequently, the damped natural period are not constant. Moreover, it has already been shown that, even in the linear case, an algebraic solution to the power optimization problem of both $B_{P T O}$ and $T_{L}$ is not possible (Nolan et al. 2005) (Babarit et al. 2004). Therefore, the optimal couple of control parameters $T_{L}$ and $B_{P T O}$ that maximizes the power output is calculated iteratively for each sea state. Since the optimization procedure uses the results of the nonlinear FK model simulations, such a latching control strategy effectively becomes a nonlinear model-based control.

\section{Results}

\subsection{Validation of the Froude-Krylov force calculation}

It is first necessary to evaluate the correctness of the algebraic results of the pressure integral in (11). The static part of the FK integral can be validated against an alternative method to calculate the nonlinear static FK force, which can be expressed as a nonlinear stiffness depending on the volume $V_{w e t}$ of the instantaneous wetted surface. As expected, the two methods return exactly the same results.

On the other hand, the static FK force calculated with the remeshing routine approach is slightly smaller (about $2 \%$ ). 
Table 3 Percentage difference between the algebraic dynamic Froude-Krylov force using either the long wave or the McLaurin approximation and the remeshing routine approach

\begin{tabular}{|c|c|c|c|c|c|c|c|}
\hline \multicolumn{2}{|c|}{ Steepness } & 0.006 & 0.012 & 0.018 & 0.006 & 0.012 & 0.018 \\
\hline$T_{w}(\mathrm{~s})$ & $\lambda / D$ & \multicolumn{3}{|c|}{ Long wave approximation (\%) } & \multicolumn{3}{|c|}{ McLaurin approximation (\%) } \\
\hline 3 & 2.8 & 19.7 & 20.0 & 20.3 & 0.9 & 1.2 & 1.4 \\
\hline 4 & 5.0 & 10.7 & 11.0 & 11.2 & 1.3 & 1.4 & 1.5 \\
\hline 5 & 7.8 & 5.7 & 5.9 & 5.9 & 1.6 & 1.5 & 1.5 \\
\hline 6 & 11.2 & 3.5 & 3.6 & 3.6 & 1.7 & 1.7 & 1.7 \\
\hline 7 & 15.4 & 2.5 & 2.6 & 2.8 & 1.6 & 1.7 & 1.8 \\
\hline 8 & 20.0 & 2.1 & 2.2 & 2.4 & 1.8 & 1.9 & 2.1 \\
\hline 9 & 25.2 & 1.9 & 2.1 & 2.3 & 1.8 & 2.0 & 2.2 \\
\hline 10 & 31.2 & 1.8 & 2.0 & 2.2 & 1.8 & 2.0 & 2.2 \\
\hline
\end{tabular}

The main reason of the difference is the actual geometry simulated: while the algebraic solution is based on an ideal sphere, the remeshing routine model is based on a discretized geometry, which rounds down the wetted surface and, consequently, $V_{\text {wet }}$. In fact, the nonlinear stiffness approach, based on the same discretized geometry, returns a better agreement with the remeshing approach. While the non-ideal geometry approximation is assessable only for the static part, it affects both the static and the dynamic FK forces.

The algebraic dynamic FK force can be calculated either using the long wave approximation or the McLaurin expansion, as discussed in Sect. 4. Within the expansion, all the sine terms integrate to zero, so only the cosine terms contribute to the algebraic solution. Using only the first three terms (i.e. the long wave approximation plus other two terms of the expansion), an acceptable accuracy is achieved for all the sea states in Table 2, including the shortest wave. While the algebraic solution of the dynamic FK force using the long wave approximation is shown in Table 1, Eq. (25) shows the algebraic solution of the dynamic FK integral of (17), using the first three terms of the McLaurin approximation (Table 3).

$$
\begin{aligned}
F_{F K_{d y z}}^{M c}= & \int_{0}^{2 \pi} \int_{\sigma_{1}}^{\sigma_{2}}\left(\rho g a \mathrm{e}^{\chi \sigma}(\cos \omega t+\chi f(\sigma) \cos \theta \sin \omega t\right. \\
& \left.-\frac{\chi f(\sigma) \cos \theta^{2}}{2} \cos \omega t\right) f^{\prime}(\sigma) f(\sigma) \mathrm{d} \sigma \mathrm{d} \theta \\
= & -\frac{2 \pi}{\chi} \rho g a \cos \omega t\left[\mathrm{e}^{\chi \sigma}\left(z_{d}+\frac{1}{\chi}-\sigma\right)\right]_{\sigma_{1}}^{\sigma_{2}} \\
& -\frac{\pi}{2} \chi \rho g a \cos \omega t\left[\mathrm { e } ^ { \chi \sigma } \left(z_{d} R^{2}-z_{d}^{3}+\frac{R^{2}-3 z_{d}^{2}}{\chi}\right.\right. \\
& \left.\left.-\frac{6 z_{d}}{\chi^{2}}-\frac{6}{\chi^{3}}\right)\right]_{\sigma_{1}}^{\sigma_{2}} \\
& -\frac{\pi}{2} \chi \rho g a \cos \omega t\left[\mathrm { e } ^ { \chi \sigma } \left(\sigma \left(3 z_{d}^{2}-R^{2}+\frac{6 z_{d}}{\chi}\right.\right.\right. \\
& \left.\left.\left.+\frac{6}{\chi^{2}}\right)-\sigma^{2}\left(3 z_{d}+\frac{3}{\chi}\right)+\sigma^{3}\right)\right]_{\sigma_{1}}^{\sigma_{2}}
\end{aligned}
$$

where the notation is the same as that used for the spherical profile defined in Table 1.

Table 3 shows the percentage difference between the dynamic FK force calculated using the two algebraic methods and the remeshing routine approach. As for the static FK force, the dynamic force is always smaller in the remeshed approach, mainly because of the discretized geometry approximation. For long waves, the two algebraic methods are significantly overlapping and the small difference with the remeshing approach is mainly due to the geometry approximation. Conversely, when short waves are considered, only the McLaurin method is effective. Finally, in addition to a dependence on the wave length, the relative error slightly increases when the wave steepness increases.

\subsection{Results}

Section 6.1 shows that, geometry approximation apart, the results of the algebraic approach are essentially the same as the remeshing routine approach. Nevertheless, Table 4 shows that the computational time of the algebraic nonlinear model is similar to the linear model, while the remeshing routine is several times slower.

Hereafter, referring to Table 2, only the highest wave steepness (0.018) is considered to compute the response of the device, since it generates the most significant nonlinear behaviour. The difference in amplitude of motion between the linear and nonlinear FK model is analyzed through the response amplitude operator (RAO). Figure 4a shows that,

Table 4 Normalized computational time of each nonlinear FK model to linear model

Normalized computational time

Algebraic method

Long wave approximation $\quad 1.35$

McLaurin approximation $\quad 1.65$

Remeshing method $\quad 5.15$ 


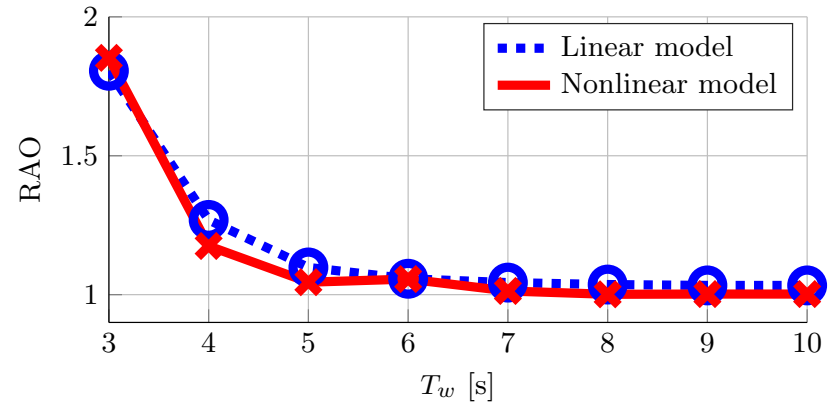

(a)

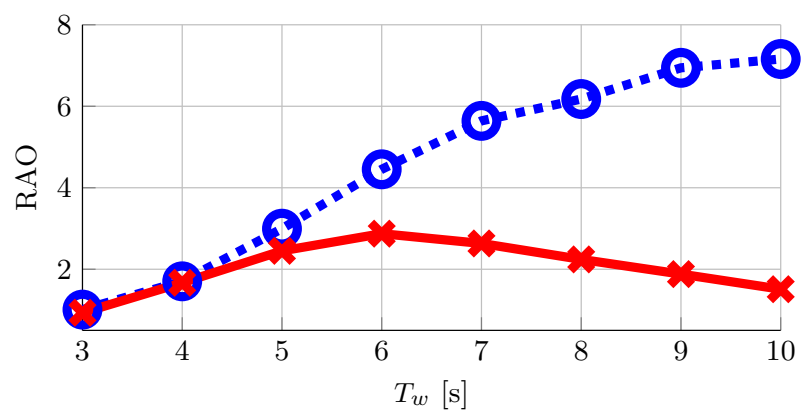

(b)

Fig. 4 Response amplitude operator (RAO) of linear and nonlinear model without control (a) and with control (b)

without the controller, the responses are very similar. In fact, the motion of the device is amplified at frequencies close to resonance, but the corresponding wave heights are small. On the other hand, bigger waves occur at frequencies far from resonance, when the device acts like a wave follower. In both cases, the variation of instantaneous wetted surface is limited, therefore, nonlinearities have little relevance.

In contrast, latching control increases the relative motion between the device and the wave, enhancing the influence of nonlinearities. Consequently, as shown by the RAO in Fig. $4 \mathrm{~b}$, the linear model significantly overestimates the response of the device at large periods when the wave height is bigger.

Comparing Fig. 4a, b, it is evident that the impact of nonlinear FK forces is enhanced by the controller since, as shown in Fig. 5, they magnifies the amplitude of the motion with respect to the free surface elevation and, as a consequence, the change in instantaneous wetted surface. Without control, the linear and nonlinear models significantly overlap, since the relative displacement is small. Conversely, when the controller is applied, the relative displacement is augmented and the linear model diverges from the nonlinear. Nevertheless, the finite geometry of the floater imposes a physical limit, since a relative displacement larger than the draft would mean that the body is completely out of the water. In such a situation, all hydrodynamic forces should be null, so the body is pulled back into the water by the gravity force.

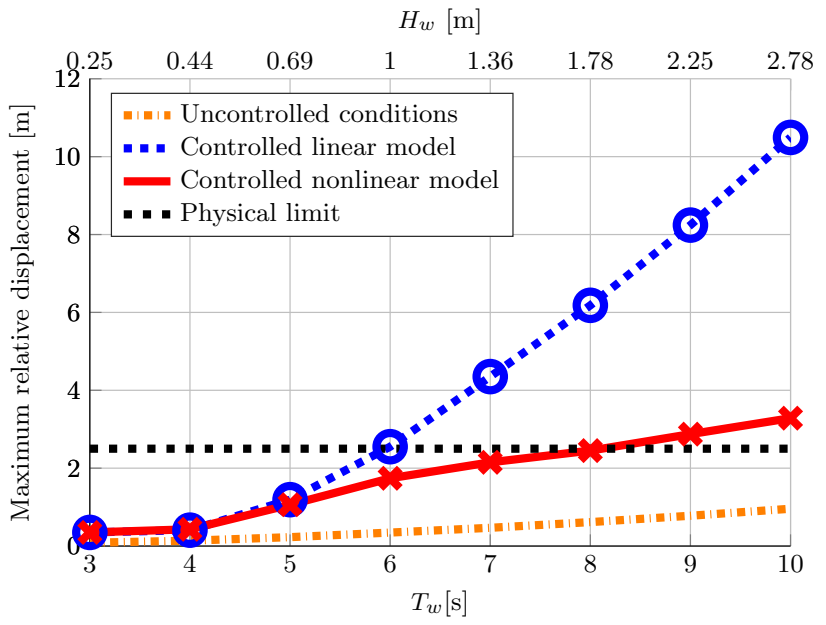

Fig. 5 Maximum relative displacement between the vertical displacement of the floater and the free surface elevation for linear and nonlinear model under uncontrolled and controlled conditions. The physical limit represents the relative displacement equal to the draft

On the contrary, all hydrodynamic forces in the linear model are computed with respect to the mean wetted surface, which is, therefore, unrealistic when large motions occur. However, the nonlinear FK model takes into account the instantaneous wetted surface, giving realistic FK forces, while the diffraction force is calculated as in the linear model. As a result, the linear model crosses the physical threshold (relative displacement greater then the draft) and becomes unrealistic for a wave much smaller than for the nonlinear FK model.

The relative comparison between linear and nonlinear models strongly depends on the device geometry; in the case of the sphere, the nonlinear FK forces are smaller than the linear FK forces because the area of the intersection between the instantaneous wetted surface and the free surface elevation is always smaller than the CSA at the still water level. If a cylinder was considered, nonlinear and linear forces would be the same (Peñalba et al. 2015b). Conversely, if the CSA increases as the device moves away from the mean position, the nonlinear FK forces would be larger than the linear. Nevertheless, to the best of knowledge of the authors, all existing point absorbers have decreasing diameter with draft and the vast majority are either spheres or cylinders. In general, if the area increases with draft, the normal to the surface will be upwards; eventually the normal must change sign and point downwards as the draft increases. The consequence is that the Froude-Krylov forces on the surface would partially cancel out, generating a small resulting force on the device. Clearly such a situation is not suitable for a wave energy converter, since the main objective is to excite the body rather than stabilize it.

For each sea state, the optimal latching control parameters $B_{P T O}$ and $T_{L}$ are defined iteratively, thanks to the low computational cost of both models. Figure 6 shows an example 


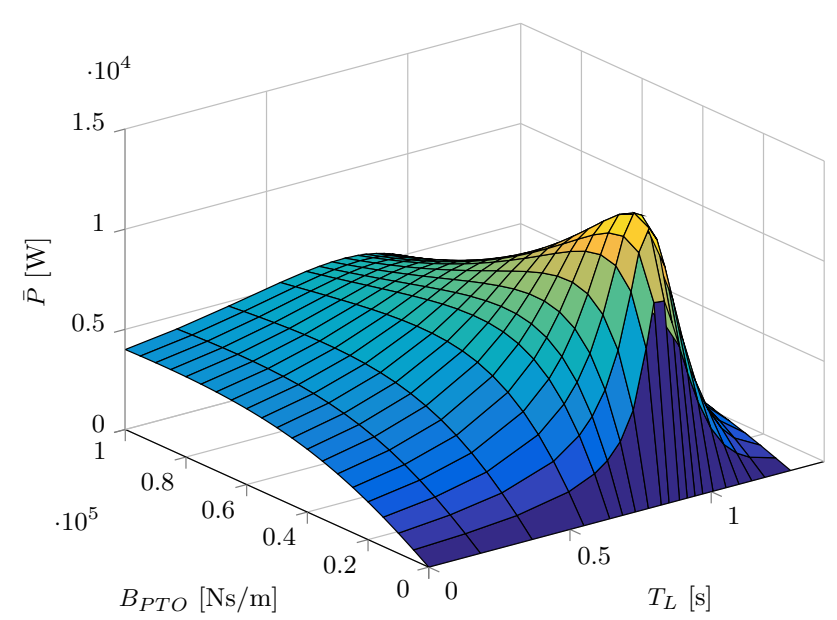

Fig. 6 Power graph for different control parameters (damping coefficient $B_{P T O}$ and latching duration $T_{L}$ ), using the nonlinear model for the wave with period $T_{w} 5 \mathrm{~s}$

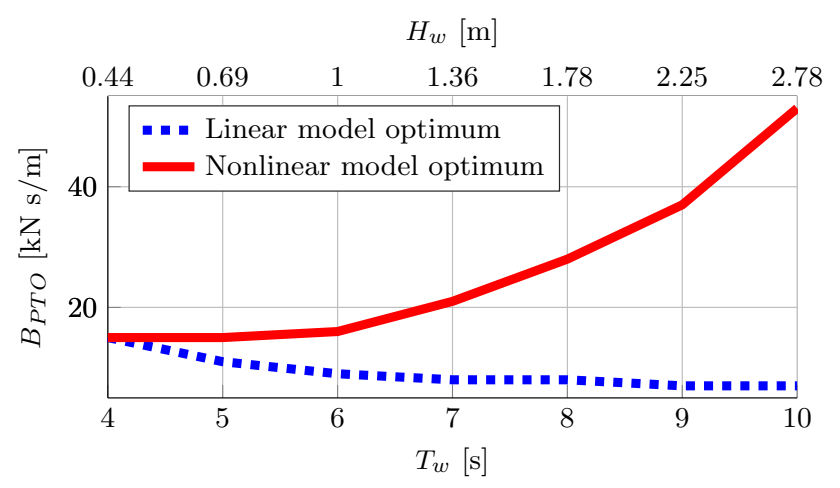

Fig. 7 Optimal profiles of PTO damping coefficients $B_{P T O}$ for linear and nonlinear models

of the power graph used to identify the point of maximum power.

The resulting optimal profiles of the control parameters for each sea state are presented in Figs. 7 and 8. In the linear model, the progressive reduction of the optimal damping as $T_{w}$ increases depends on the reduction of the radiation damping term of the sphere at low frequencies (Falnes 2002). Conversely, the optimal $B_{P T O}$ for the nonlinear model increases. As a consequence, the damped natural period $T_{d}$ of the nonlinear model is longer and the optimal latching duration is shorter; Fig. 8 presents the algebraic optimum for the nonlinear model (dash-dot red line), computed using (24) with the optimal $B_{P T O}$ of Fig. 7. Nevertheless, the natural period $T_{n}$ used in (24) is accurate only for small amplitudes of motion, since it is calculated from the mean cross sectional area. On the other hand, the variations in instantaneous wetted surface considered in the nonlinear model result in a longer natural period and, consequently, shorter optimal latching duration (solid red line).

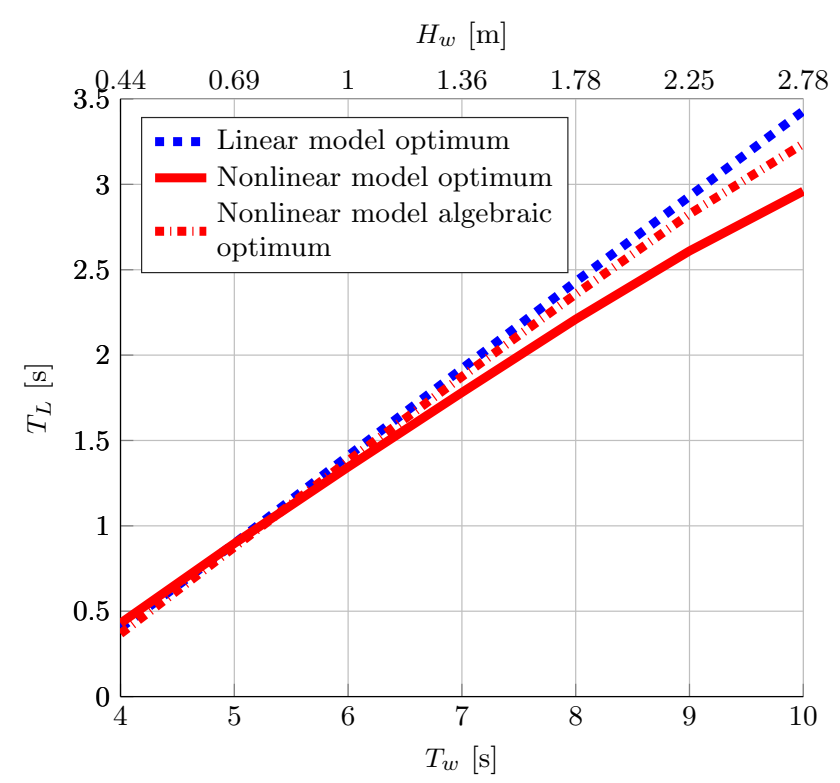

Fig. 8 Optimal profiles of latching durations $T_{L}$ for linear and nonlinear models. The algebraic optimum for the nonlinear model refers to the application of (24) with the optimal damping in Fig. 7

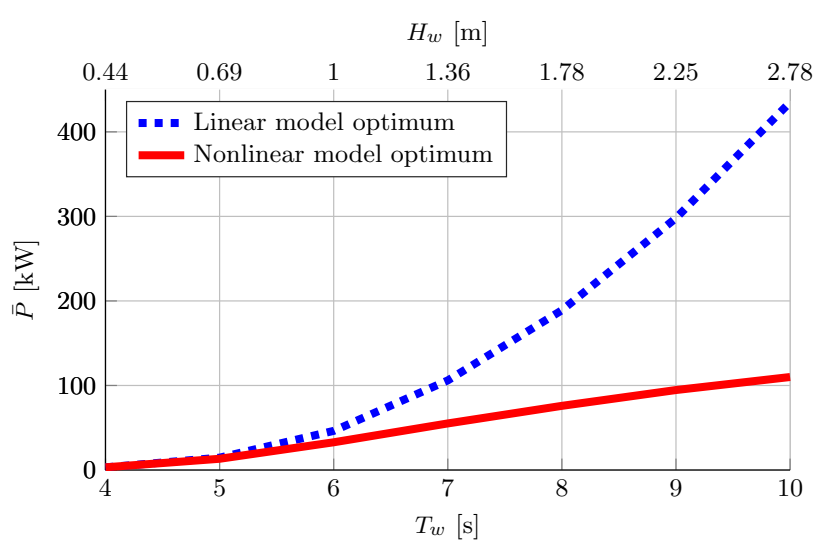

Fig. 9 Optimal power extracted using linear and nonlinear models

Figure 9 shows the mean power extracted using the optimal control parameters. Consistent with the RAO of Fig. $4 b$, the linear model overestimates the motion of the wave energy device, leading to an overly optimistic power production assessment.

\section{Conclusions}

Previous work (Merigaud et al. 2012; Peñalba et al. 2015b) showed that nonlinear Froude-Krylov forces are likely to be the dominant source of nonlinearities for heaving point absorbers with nonuniform cross sectional area under controlled conditions. This paper proposes an algebraic formulation for nonlinear static and dynamic FK forces valid for any axisymmetric heaving wave energy converter. An alternative 
method using a remeshing of the surface is used to validate the algebraic approach; despite a considerable computational saving, the nonlinear FK forces are computed with the same accuracy.

From the comparison between linear and algebraic nonlinear model applied to a spherical point absorber subject to linear regular waves, the following conclusions are drawn:

- Nonlinear FK forces are relevant only when the instantaneous wetted surface experiences substantial variations. Under latching control conditions, the amplitude of motion is exaggerated and the behavior as a wave follower prevented. Likewise, other control philosophies (apart from latching) might exaggerate the body motion relative to the free surface elevation, causing significant changes in the nonlinear FK forces (Bacelli and Ringwood 2015).

- Nonlinearities affect the choice of optimal latching control parameters: the required PTO damping coefficient $B_{P T O}$ is considerably higher than in the linear model. Furthermore, the damped natural period is longer, due to both the higher damping and the changing of the instantaneous cross sectional area, so the optimal latching duration $T_{L}$ is smaller.

- The linear model leads to an overly optimistic power production assessment.

The study carried out in this paper focuses on monochromatic sea states to understand the behavior of the system at each single frequency. Nevertheless, a further step toward a more realistic situation will be to include panchromatic waves. In the power production region for WECs, the vast majority of waves are linear, so the total pressure of an irregular sea state can be calculated as the superposition of regular components. Then, the instantaneous wetted surface is determined by the irregular free surface elevation and the displacement of the device. The higher the number of frequency components, the higher the computational effort required in the algebraic calculation of nonlinear FK forces. Depending on the ratio of the wavelength to the device dimension, either the long wave approximation or the McLaurin expansion is chosen for each wave component to invest the least computational effort to satisfy a certain accuracy target. However, the computational saving of the algebraic method compared to the remeshing approach, may not scale up in the same way for polychromatic waves. The computational time of the remeshing approach is mainly influenced by the remeshing routine used to determine the instantaneous wetted surface, which requires the same computational effort both in regular and irregular sea states, while the computational effort of the algebraic method increases (linearly) with the number of wave frequencies.
Acknowledgements This paper is based upon work supported by Science Foundation Ireland under Grant No. 13/IA/1886.

\section{References}

Agamloh EB, Wallace AK, Von Jouanne A (2008) Application of fluidstructure interaction simulation of an ocean wave energy extraction device. Renew Energy 33(4):748-757

Babarit A (2010) Achil3D v2.011 User Manual. Laboratoire de Mecanique des Fluides CNRS, Ecole Central de Nantes

Babarit A, Clément A (2006) Optimal latching control of a wave energy device in regular and irregular waves. Appl Ocean Res 28:77-91

Babarit A, Duclos G, Clément A (2004) Comparison of latching control strategies for a heaving wave energy device in random sea. Appl Ocean Res 26(5):227-238

Babarit A, Mouslim H, Clément A, Laporte-Weywada P (2009) On the numerical modelling of the non linear behaviour of a wave energy converter. In: ASME 2009 28th international conference on ocean, offshore and arctic Engineering, American Society of Mechanical Engineers, Honolulu, pp 1045-1053

Bacelli G, Ringwood JV (2015) Numerical optimal control of wave energy converters. Sustain Energy IEEE Trans 6(2):294-302

Budal K, Falnes J, Kyllingstad A, Oltedal G (1979) Experiments with point absorbers. In: Proceedings of first symposium on wave energy utilization. Gothenburg, Sweeden, pp 253-282

Clement A, Ferrant P (1988) Nonlinear water waves: IUTAM Symposium, Tokyo Japan, August 25-28, 1987. Superharmonic waves generated by the large amplitude heaving motion of a submerged body. Springer, Berlin, pp 423-433

Cummins W (1962) The impulse response function and ship motion. Schiffstechnik 9:101-109

Falnes J (2002) Ocean waves and oscillating systems. Cambridge University Press, Cambridge

Gilloteaux JC (2007) Mouvements de grande amplitude d'un corps flottant en fluide parfait. application à la récupération de l'énergie des vagues. $\mathrm{PhD}$ Thesis, Ecole Centrale de Nantes-ECN

Giorgi G, Ringwood JV (2016a) Implementation of latching control in a numerical wave tank with regular waves. J Ocean Eng Mar Energy 2(2):211-226

Giorgi G, Ringwood JV (2016b) Nonlinear Froude-Krylov force representations for heaving buoy wave energy converters. In: Submitted to the 3rd Asian wave and tidal energy conference, Singapore

Giorgi G, Ringwood JV (2016c) Nonlinear hydrodynamic force relevance for different wave energy converter types. In: Submitted to the 3rd Asian wave and tidal energy conference, Singapore

Guerinel M, Zurkinden AS, Alves M, Sarmento AJ (2013) Validation of a partially nonlinear time domain model using instantaneous froude-krylov and hydrostatic forces. In: 10th Ewtec 2013 European wave and tidal energy conference Series, Technical Committee of the European Wave and Tidal Energy Conference, Aalborg, Denmark

Hansen RH, Kramer MM (2011) Modelling and control of the wavestar prototype. In: Proceedings of the 11th European Wave and Tidal Energy Conference, Southampton, UK

Henry A, Schmitt P, Whittaker T, Rafiee A, Dias F, et al. (2013) The characteristics of wave impacts on an oscillating wave surge converter. In: The twenty-third international offshore and polar engineering conference, International Society of Offshore and Polar Engineers, Anchorage, Alaska, pp 101-110

Mehaute BL (1976) An introduction to hydrodynamics and water waves. Springer, Berlin

Merigaud A, Gilloteaux JC, Ringwood JV (2012) A nonlinear extension for linear boundary element methods in wave energy device modelling. In: ASME 2012 31st international conference on ocean, 
offshore and arctic engineering, American Society of Mechanical Engineers, Rio de Janeiro, Brazil, pp 615-621

Morison JR, Johnson JW, Schaaf SA (1950) The force exerted by surface waves on piles. J Pet Technol 2(5):149-154

Nolan G, Ringwood J, Leithead W, Butler S (2005) Optimal damping profiles for a heaving buoy wave-energy converter. In: Proceedings of the fifteenth international offshore and polar engineering conference (ISOPE), vol 1. Seoul, Korea, pp 477-485

Omidvar P, Stansby PK, Rogers BD (2012) Wave body interaction in 2D using smoothed particle hydrodynamics $(\mathrm{SPH})$ with variable particle mass. Int J Num Methods Fluids 68(6):686-705

Peñalba M, Giorgi G, Ringwood JV (2015a) A review of non-linear approaches for wave energy converter modelling. In: Proceedings of European Wave and Tidal Energy Conference, Nantes, France

Peñalba M, Merigaud A, Gilloteaux JC, Ringwood JV (2015b) Nonlinear Froude-Krylov force modelling for two heaving wave energy point absorbers. In: Proceedings of European Wave and Tidal Energy Conference, Nantes, France
Rafiee A, Dias F (2013) Two-dimensional and three-dimensional simulation of wave interaction with an oscillating wave surge converter. In: Proceedings of 28th international workshop on water waves and floating bodies, L'isle sur la Sourge, France

Ringwood JV, Butler S (2004) Optimisation of a wave energy converter. In: Proceedings of IFAC conference on control applications in marine systems, Ancona, Italy

Taghipour R, Perez T, Moan T (2007) Hybrid frequency-time domain models for dynamic response analysis of marine structures. Ocean Eng 35(7):685-705 